\title{
Composition chimique et coagulation du lait sécrété en fin de gestation et au début de la lactation suivante par des vaches laitières conduites sans période sèche
}

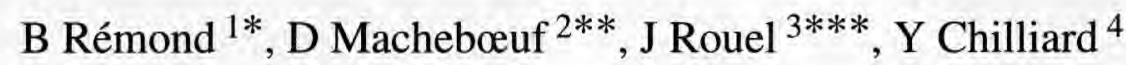

${ }^{\prime}$ Laboratoire adaptation des herbivores aux milieux, Inra, Theix, 63122 Saint-Genès-Champanelle;

${ }^{2}$ Laboratoire de recherches fromagères, Inra, 36, rue de Salers, 15000 Aurillac ;

${ }^{3}$ Domaine du Roc, Inra, 63210 Orcival ; ${ }^{4}$ Laboratoire de sous-nutrition des ruminants, Inra, Theix, 63122 Saint-Genès-Champanelle, France

(Reçu le 21 janvier 1997 ; accepté le 6 mai 1997)

\begin{abstract}
Summary - Chemical composition and clotting properties of milk secreted during late pregnancy by dairy cows managed without the dry period. Among 12 dairy Holstein cows in their first lactation that we attempted to milk until their 2 nd calving, five had to be dried off, 9 days before calving on average. Milk progressively enriched in somatic cells and fat. Increase in proteins was mainly due to caseins but soluble proteins increased relatively more, so that the casein/protein ratio decreased. Increase in IgG1 explained $80 \%$ of the soluble protein augmentation. Free fatty acids measured either in the milk just milked or after $26 \mathrm{~h}$ in refrigerator highly increased in late pregnancy. $\mathrm{pH}$ collapsed just before calving (6.22). Rennet clotting time (Ri) and curd firmness time (K20i) measured at natural $\mathrm{pH}$ were divided by a nearly 3 factor whereas curd firmness (A30i) increased by approximately $40 \%$. Measured at standardized $\mathrm{pH}(6.60)$ these clotting properties changed similarly but in an attenuated manner. In the succeeding lactation, secretion of milk by these cows was lower than that by the control cows $(n=8)$ conventionally dried off in the late preceding lactation, but their milk was richer in proteins.
\end{abstract}

dairy cow / late pregnancy / milk composition / clotting property

Résumé - Sur 12 vaches primipares Holstein destinées à être traites jusqu'au deuxième vêlage, 7 ont effectivement pu l'être et 5 ont dû être taries en moyenne 9 jours avant le vêlage. Le lait s'est progressivement enrichi en cellules somatiques et en matières grasses. L'accroissement de la teneur en protéines a été majoritairement dû aux caséines, mais les protéines solubles ont relativement plus augmenté que les caséines, de sorte que la proportion des caséines dans les protéines a diminué. L'accrois-

Adresses actuelles : *École nationale d'ingénieurs des travaux agricoles, Marmilhat, 63370 Lempdes ; **Station de recherches sur la nutrition des herbivores, Inra, Theix, 63122 SaintGenès-Champanelle ; ***Laboratoire de recherche sur la sous-nutrition des ruminants, Inra, Theix, 63122 Saint-Genès-Champanelle, France 
sement des immunoglobulines G1 a expliqué $80 \%$ de celui des protéines solubles. La teneur en acides gras libres dans le lait natif et après 26 heures au réfrigérateur a fortement augmenté en fin de gestation. Le pH du lait s'est effondré à l'approche du vêlage $(6,22)$ et les temps de coagulation (Ri) et de raffermissement (K20i) ont été divisés par un facteur proche de 3, tandis que la fermeté du gel s'accroissait d'environ $40 \%$. Mesurées à $\mathrm{pH}$ standardisé $(6,60)$, ces caractéristiques de coagulation ont évolué de façon semblable, mais atténuée. Au cours de la lactation suivante, la sécrétion de lait de ces animaux a été plus faible que celle d'animaux témoins $(n=8)$ taris environ 2 moís à la fin de la lactation précédente, mais leur lait a été plus riche en protéines.

vache laitière / fin de gestation / composition du lait / coagulation du lait

\section{INTRODUCTION}

Les variations de la composition du lait et, dans une moindre mesure, de son aptitude à la coagulation au cours du cycle normal de lactation sont assez bien connues (Rémond, 1987; Martin et Coulon, 1995). Elles le sont en revanche très peu (voir ci-après) au cours des 2 derniers mois de gestation lorsque les vaches sont traites sans interruption jusqu' au vêlage suivant. Cette conduite du cycle de production n'était, en effet, pas du tout utilisée par les éleveurs car elle déprime fortement ( 25 à $40 \%$ ) la production laitière au cours de la lactation suivante (Klein et Woodward, 1943 ; Swanson, 1965 ; Rémond et al, 1992).

La politique des quotas laitiers a rendu beaucoup plus lâche qu'auparavant le lien entre les performances individuelles des vaches et le revenu des éleveurs. Son instauration permet donc à ces derniers de s'intéresser à des conduites susceptibles de faciliter la conduite de leur troupeau (tarissement plus facile, alimentation plus régulière et plus économe en céréales, incidence moindre des accidents sanitaires...) et d'améliorer la qualité du lait (teneur en protéines : Rémond et al, 1992), même au prix d'une certaine diminution des performances individuelles des animaux. L'omission de la période sèche ou, plus vraisemblablement, la réduction de sa durée méritent donc d'être réévaluées car elles permettent de satisfaire une partie de ces souhaits. Il est cependant essentiel d'en connaître les conséquences sur la qualité du lait.

La composition chimique du lait sécrété en fin de gestation a fait l'objet de très peu d'études. Toutefois, Rémond et al (1992) et Rémond et Bonnefoy (1997) ont montré que la teneur en caséines s'accroissait proportionnellement moins que celle des protéines solubles à l'approche du vêlage, de sorte que la proportion des caséines dans les protéines totales diminuait. Par ailleurs, Chilliard et al (1989) et Rémond et Bonnefoy (1997) ont observé des accroissements très forts, mais très variables entre les animaux de la concentration des acides gras libres. En revanche, aucune étude, à notre connaissance, n'a visé à mieux connaître l'aptitude à la coagulation du lait produit pendant cette période, alors que la fabrication fromagère est la principale utilisation du lait dans notre pays.

Dans une étude où nous avons essayé d'omettre la période sèche au cours de trois lactations consécutives, nous avons décrit, au cours de la première année d'expérimentation, l'évolution détaillée des principaux constituants du lait, ainsi que celle de son aptitude à la coagulation.

\section{MATÉRIEL ET MÉTHODES}

\section{Schéma expérimental et conduite des animaux}

Au cours de l'été, 23 vaches Holstein en première lactation ayant vêlé en moyenne à la fin 
du mois d'octobre (du 30 septembre au 26 décembre), à l'âge de 34 mois, ont été réparties en deux lots de caractéristiques semblables (production laitière, âge, date de vêlage, poids vif), mais de tailles différentes. Un lot était destiné à être tari environ 2 mois avant le deuxième vêlage ( $\operatorname{lot} \mathrm{T} ; n=9$ ) et l'autre lot devait être trait jusqu'au vêlage suivant (lot NT ; $n=14$ ), ou aussi longtemps que la production laitière journalière était d'au moins $4 \mathrm{~kg} / \mathrm{j}$. Les animaux qui étaient au pâturage ont été rentrés à l'étable, en stabulation entravée, au moins 3 semaines avant la date prévue de leur deuxième vêlage. Pendant la fin de la gestation, ils ont reçu de l'ensilage d'herbe à volonté et de l'aliment concentré en quantité croissante au cours des 3 dernières semaines $(1,2$ puis $3 \mathrm{~kg} / \mathrm{j})$. A partir du vêlage, il ont reçu à volonté une ration complète composée, sur la base de la matière sèche, de $56 \%$ d'ensilage d'herbe, $15 \%$ de foin de regain, $28 \%$ d'aliment concentré de production et $1 \%$ de complément minéral vitaminé. Les vaches étaient traites dans une salle de traite à $6 \mathrm{~h}$ et à $17 \mathrm{~h}$.

\section{Mesures}

La quantité de lait produite a été pesée à chaque traite. Le lait de la traite du matin a été échantillonné une fois au cours du $7^{\mathrm{e}}$ mois de gestation (deux lots), une fois toutes les 2 semaines au cours des $8^{\mathrm{e}}$ et $9^{\mathrm{e}}$ mois (lot NT) puis, après le vêlage, à 2 reprises : au cours de la $2^{\mathrm{e}}$ semaine de la lactation et entre les $9^{\mathrm{e}}$ et $12^{\mathrm{e}}$ semaines de lactation (lots $\mathrm{T}$ et NT). Une fraction $(50 \mathrm{~mL})$ a été immédiatement réfrigérée à $4{ }^{\circ} \mathrm{C}$ et expédiée par colis express dans un emballage isotherme au laboratoire Inra d'Aurillac, en vue de la détermination du $\mathrm{pH}$ et des caractéristiques de coagulation, à l'aide d'un appareil Formagraph : le temps de coagulation ( $\mathrm{R}$ exprimé en minutes), le temps de raffermissement (K20 exprimé en minutes) et la fermeté du gel mesurée 30 minutes après l'emprésurage (A30 exprimé en millimètres). Les mesures ont été faites le lendemain matin, dans le lait natif ( $\mathrm{Ri}, \mathrm{K} 20 \mathrm{i}, \mathrm{A} 30 \mathrm{i})$ et après l'ajustement de son pH à 6,60 (Rs, K20s, A30s), selon le protocole décrit par Machebœuf et al (1993). La température du lait à l'arrivée au laboratoire était de $8,2^{\circ} \mathrm{C}$ en moyenne (maximum $\left.12,0^{\circ} \mathrm{C}\right)$. Une fraction du lait $(50 \mathrm{~mL})$ a été congelée telle quelle dès la traite, en vue du dosage de l'azote total, de l'azote soluble et de l'azote non protéique (Rowland, 1938) et des immunoglobulines G1 (Levieux, 1991). Deux autres fractions (de $18 \mathrm{~mL}$ chacune) ont été congelées après thermisation du lait à $65^{\circ} \mathrm{C}$ pendant 30 minutes, effectuée soit juste après la traite, soit après 26 heures au réfrigérateur, en vue du dosage des acides gras libres (Chazal et Chilliard, 1986). Ce dosage n'a pas pu être fait dans six des huit échantillons prélevés à la période 4 , car les laits étaient coagulés et n'ont pas pu être remis en solution. Dans une dernière fraction $(50 \mathrm{~mL})$, les teneurs en matières grasses et en lactose ont été mesurées par spectrophotométrie infrarouge et les cellules somatiques par comptage automatique (appareils Milkoscan 605 et Fossomatic ; Foss Electric, Hillerod, Danemark).

\section{Analyse des résultats}

Les résultats ont été répartis en six périodes, selon la durée séparant le prélèvement du vêlage : quatre périodes avant le vêlage $(1:>50$ jours ; 2 : 50 à 31 jours ; $3: 30$ à 11 jours ; $4: 10$ jours au vêlage) et deux périodes après. Les analyses de variance et covariance ont été réalisées avec la procédure Proc Mixed (SAS, 1992) et ont porté séparément d'une part sur les données (ou leur logarithme) enregistrées sur le lot NT avant le vêlage (quatre périodes) et d'autre part sur les données enregistrées dans les deux lots après le vêlage (deux périodes). Dans l'analyse des données enregistrées après le vêlage, les résultats de la période 1 avant le vêlage ( $>50$ jours) ont été utilisés comme covariable. Les résultats portent sur 19 vaches ( 83 données), 2 vaches de chaque lot ayant été éliminées pour des raisons sanitaires indépendantes de l'expérimentation. Pour les quatre périodes de fin de gestation et de lactation (lot NT), nous avons vérifié que les résultats calculés avec les seules vaches présentes en période 4 étaient semblables à ceux portant sur tous les animaux, bien que parfois moins significatifs, probablement à cause du plus faible nombre de données.

\section{RÉSULTATS}

\section{Production et composition du lait}

Parmi les 12 vaches du lot NT conservées dans le dépouillement des résultats, 7 ont pu être traites jusqu'au vêlage et 5 ont été 
Tableau I. Production et composition du lait (moyennes ajustées).

Milk production and composition (adjusted means).

\begin{tabular}{|c|c|c|c|c|c|c|c|c|c|c|c|}
\hline \multirow{3}{*}{$\begin{array}{l}\text { Jours avant (-) ou après le vêlage } \\
\text { Lot } \\
\text { Nbre de vaches }\end{array}$} & \multicolumn{4}{|c|}{ Avant le vêlage } & \multicolumn{4}{|c|}{ Après le vêlage } & \multicolumn{3}{|c|}{ Analyse statistique } \\
\hline & \multirow{2}{*}{$\begin{array}{c}<-50 \\
N T \\
8\end{array}$} & \multicolumn{2}{|c|}{$-50 \grave{a}-31-30 \grave{a}-11$} & \multirow{2}{*}{$\begin{array}{c}-10 \grave{a} 0 \\
N T \\
7\end{array}$} & \multicolumn{2}{|c|}{$6 \grave{a} 18$} & \multicolumn{2}{|c|}{57 à 82} & \multirow{2}{*}{$\begin{array}{l}\text { Avant } \\
\text { vêlage } \\
\text { Période }\end{array}$} & \multicolumn{2}{|c|}{ Après vêlage } \\
\hline & & $\begin{array}{c}N T \\
11\end{array}$ & $\begin{array}{c}N T \\
11\end{array}$ & & $\begin{array}{l}T \\
7\end{array}$ & $\begin{array}{l}N T \\
12\end{array}$ & $\begin{array}{l}T \\
6\end{array}$ & $\begin{array}{c}N T \\
12\end{array}$ & & Lot & Période \\
\hline Lait (kg/jour) & $9,9 \mathrm{a}$ & $8,4 \mathrm{~b}$ & $7,0 \mathrm{c}$ & $4,4 d$ & $28,9 \mathrm{a}$ & $21,6 \mathrm{~b}$ & $30,1 \mathrm{a}$ & $23,9 \mathrm{~b}$ & $<0,01$ & $<0,01$ & ns \\
\hline Lactose $(\mathrm{g} / \mathrm{kg})$ & 46,3 & 46,2 & 46,8 & 44,0 & $49,2 \mathrm{ab}$ & $47,7 \mathrm{a}$ & $49,6 a b$ & $50,5 \mathrm{~b}$ & ns & ns & $<0,05$ \\
\hline Cellules $(\times 1000)$ & 144ab & $164 a$ & $235 b$ & $407 c$ & 189 & 175 & 194 & 98 & $<0,01$ & ns & ns \\
\hline Protéines (g/kg) & $37,3 a$ & $39,3 \mathrm{ab}$ & $42,9 b$ & $55,1 \mathrm{c}$ & $34,8 \mathrm{a}$ & $38,7 \mathrm{~b}$ & $29,2 \mathrm{c}$ & $33,4 \mathrm{ad}$ & $<0,01$ & $<0,01$ & $<0,01$ \\
\hline Caséines $(\mathrm{g} / \mathrm{kg})$ & $31,2 \mathrm{a}$ & $31,8 \mathrm{ab}$ & $34,8 b$ & $42,5 \mathrm{c}$ & $28,6 \mathrm{ab}$ & $31,1 \mathrm{a}$ & $23,4 \mathrm{c}$ & $26,9 b$ & $<0,01$ & $<0,05$ & $<0,01$ \\
\hline Protéines solubles $(\mathrm{g} / \mathrm{kg}$ ) & $6,4 \mathrm{ab}$ & $7,2 \mathrm{ac}$ & $8,2 \mathrm{c}$ & $12,3 \mathrm{~d}$ & $5,9 \mathrm{ab}$ & $6,5 \mathrm{a}$ & $5,3 \mathrm{c}$ & $5,5 \mathrm{bc}$ & $<0,01$ & ns & $<0,01$ \\
\hline Immunoglobulines $\mathrm{G} 1$ ( $\mathrm{g} / \mathrm{kg}$ ) & $0,64 \mathrm{a}$ & $0,85 b$ & $1,52 \mathrm{c}$ & $5,30 \mathrm{~d}$ & $1,0 \mathrm{ab}$ & $1,0 \mathrm{a}$ & $0,4 \mathrm{c}$ & $0,6 \mathrm{bd}$ & $<0,01$ & ns & $<0,01$ \\
\hline Caséines \% protéines & $83,2 \mathrm{a}$ & $81,2 \mathrm{ab}$ & $81,3 b$ & $77,9 \mathrm{c}$ & 82,8 & 82,7 & 81,6 & 83,0 & $<0,01$ & ns & ns \\
\hline Matières grasses $(\mathrm{g} / \mathrm{kg})$ & $44,6 \mathrm{ab}$ & $49,4 \mathrm{ad}$ & $52,3 \mathrm{~cd}$ & $39,9 \mathrm{bc}$ & $26,3 a$ & $40,9 b c$ & $32,0 \mathrm{ac}$ & $30,4 \mathrm{a}$ & $<0,05$ & ns & ns \\
\hline AGL-t0 (mg C16/100 g matières grasses) & $437 \mathrm{a}$ & $416 a$ & $608 \mathrm{a}$ & $1959 \mathrm{~b}$ & 669 & 537 & 618 & 562 & $<0,05$ & ns & ns \\
\hline AGL-t26 (mg C16/100 g matières grasses) & $944 \mathrm{a}$ & $1012 \mathrm{a}$ & $2039 b$ & $14765 \mathrm{c}$ & 1150 & 885 & 955 & 861 & $<0,01$ & ns & ns \\
\hline
\end{tabular}

Analyses statistiques effectuées séparément avant et après le vêlage ; les moyennes qui ne sont pas suivies de la même lettre sont différentes au seuil de signification de $1 \%$ ou $5 \%$. Les résultats de la vache ayant une teneur de lait très élevée en IgG1 à la période 4 n'ont pas été utilisés dans les analyses statistiques, pour cette période. Data before and after calving were separately analysed; means that do not share the same letter are different at the $1 \%$ or $5 \%$ level of significance. Results of the cow whose milk had a very high IgGl content at period 4 were not used, for that period, in the statistical analyses 
taries 3 à 20 jours (moyenne : 9 jours) avant le vêlage, à une production journalière moyenne de $3,5 \mathrm{~kg} / \mathrm{j}$.

Avant le vêlage, la quantité de lait sécrété a diminué et la concentration des matières grasses et des protéines a augmenté, de même que le nombre de cellules somatiques (tableau I). L'accroissement de concentration des protéines totales d'une période à la suivante a été majoritairement dû pour toutes les vaches sauf une (voir ci-après), à celui des caséines. L'accroissement de la teneur en protéines solubles pendant la même période est expliqué à $80 \%$ par celui des immunoglobulines (la liaison entre leurs accroissements a été hautement corrélée : $\left.r^{2}=0,75\right)$. Cependant, l'accroissement relatif plus élevé des protéines solubles $(+92 \%)$ que des caséines $(+36 \%)$ au cours des quatre périodes de fin de gestation a entraîné une diminution limitée ( 5 points $\%$ ) mais significative de la proportion des caséines dans les protéines.

Pour la vache qui fait exception (voir plus haut), la très forte augmentation de concentration des protéines totales entre les périodes 3 et $4(+83 \mathrm{~g} / \mathrm{kg}$ contre $+14,3 \mathrm{~g} / \mathrm{kg}$ pour les sept autres vaches) a été le fait, pour les trois quarts, de l'augmentation des protéines solubles $(+62,2 \mathrm{~g} / \mathrm{kg}$ contre $+4,6 \mathrm{~g} / \mathrm{kg}$ pour les sept autres vaches), qui était ellemême entièrement due à celle des IgG1 $(+62,1 \mathrm{~g} / \mathrm{kg}$ contre $+3,5 \mathrm{~g} / \mathrm{kg})$. Il est à noter que les six concentrations les plus élevées (supérieures à $3 \mathrm{~g} / \mathrm{kg}$ ) en IgG1 ont été observées en période 3 (une valeur) et en période 4 (cinq valeurs) et que pour cinq de ces six valeurs les quantités de lait sécrétées étaient inférieures à $5 \mathrm{~kg} / \mathrm{j}$ (fig 1 ).

L'approche du vêlage a accru la variabilité individuelle de l'ensemble des constituants du lait ; ainsi, les coefficients de variation des teneurs en matières grasses, en protéines, en lactose et en IgG sont passés de $8 \%, 7 \%, 4 \%$ et $35 \%$ respectivement en période 1 , à $24 \%, 45 \%, 20 \%$ et $175 \%$ en période 4 .

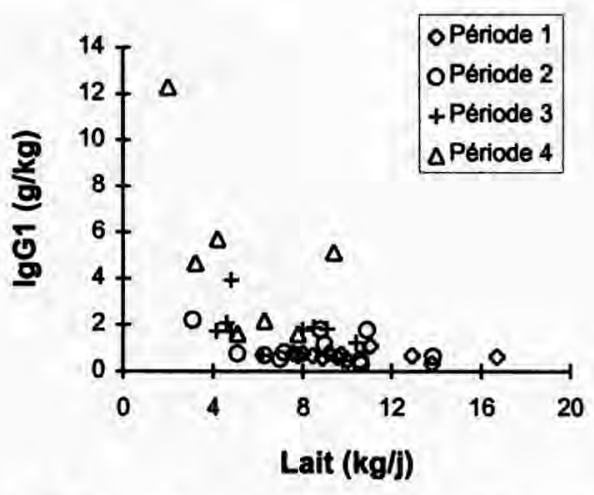

Fig 1. Liaison entre la quantité de lait sécrétée et sa teneur en IgG1 (la période-vache pour laquelle la teneur en IgGl était de $64 \mathrm{~g} / \mathrm{kg}$ a été omise). Périodes : $1:<-50$ jours avant le vêlage ; $2:-50$ à -31 jours $; 3:-30$ à -11 jours ; $4:-10$ jours au vêlage.

Relationship between the yield of milk and its IgGI content (the cow $x$ period for which the IgGl content was $64 \mathrm{~g} / \mathrm{kg}$ has been omitted). Periods : $1 ;<-50$ days before calving : $2:-50$ to -31 days $: 3:-30$ to -11 days $; 4:-10$ days to calving.

La teneur en acides gras libres initiaux (AGL-t0) a augmenté en fin de gestation. Toutefois, seule la période 4 a été différente des trois premières, car l'accroissement moyen entre les périodes 2 et 3 est imputable à une vache dont la teneur du lait en AGL-t0 était 14 fois plus élevée que celle des autres vaches (6851 vs $496 \mathrm{mg}$ d'acide gras de forme $\mathrm{C} 16 / 100 \mathrm{~g}$ matières grasses). La lipolyse dans le lait pendant son séjour de 26 heures au réfrigérateur (AGL-t26-AGLt0) a encore plus fortement augmenté que celle des AGL-t0. Les teneurs en AGL-t0 ou en AGL-t26 et la lipolyse pendant le séjour au réfrigérateur n'ont pas été liées au nombre de cellules somatiques.

La traite en fin de gestation n'a eu d'effet significatif au cours de la lactation suivante (périodes 5 et 6 ) que sur la quantité de lait produite et les teneurs en protéines et en caséines, sans cependant affecter le rapport entre ces deux fractions protéiques. 
Tableau II. pH et caractéristiques de coagulation du lait (valeurs ajustées).

pH and clotting properties of milk (adjusted means).

\begin{tabular}{|c|c|c|c|c|c|c|c|c|c|c|c|}
\hline \multirow{3}{*}{$\begin{array}{l}\text { Jours avant (-) ou après le vêlage } \\
\text { Lot } \\
\text { Nbre de vaches }\end{array}$} & \multicolumn{4}{|c|}{ Avant le vêlage } & \multicolumn{4}{|c|}{ Après le vêlage } & \multicolumn{3}{|c|}{ Analyse statistique } \\
\hline & \multirow{2}{*}{$\begin{array}{c}<-50 \\
N T \\
8\end{array}$} & \multicolumn{2}{|c|}{-50 à $-31-30 \grave{a}-11$} & \multirow{2}{*}{$\begin{array}{c}-10 \grave{a} 0 \\
N T \\
7\end{array}$} & \multicolumn{2}{|c|}{$6 \grave{a} 18$} & \multicolumn{2}{|c|}{57 à 82} & \multirow{2}{*}{$\begin{array}{l}\text { Avant } \\
\text { vêlage } \\
\text { Période }\end{array}$} & \multicolumn{2}{|c|}{ Après vêlage } \\
\hline & & $\begin{array}{c}N T \\
11\end{array}$ & $\begin{array}{l}N T \\
11\end{array}$ & & $\begin{array}{l}T \\
7\end{array}$ & $\begin{array}{l}N T \\
12\end{array}$ & $\begin{array}{l}T \\
6\end{array}$ & $\begin{array}{c}N T \\
12\end{array}$ & & Lot & Période \\
\hline $\mathrm{pH}$ natif & $6,70 \mathrm{a}$ & $6,75 \mathrm{bc}$ & $6,68 \mathrm{ac}$ & $6,22 d$ & $6,59 a$ & $6,64 a b$ & $6,66 \mathrm{~b}$ & $6,68 b$ & $<0,01$ & ns & $<0,01$ \\
\hline \multicolumn{12}{|l|}{ Mesures au pH natif } \\
\hline Temps de coagulation (min) (Ri) & $23,6 a b$ & $25,3 \mathrm{a}$ & $20,3 b$ & $7,5 \mathrm{c}$ & 16,7 & 19,4 & 20,4 & 20,7 & $<0,01$ & ns & ns \\
\hline Temps de raffermissement $(\mathrm{min})(\mathrm{K} 20 \mathrm{i})$ & $9,6 \mathrm{ab}$ & $9,5 \mathrm{a}$ & $7,1 \mathrm{~b}$ & $2,2 \mathrm{c}$ & $7,8 \mathrm{a}$ & $7,9 a$ & $18,7 \mathrm{~b}$ & $11,9 \mathrm{a}$ & $<0,01$ & ns & $<0,01$ \\
\hline Fermeté du gel $(\mathrm{mm})(\mathrm{A} 30 \mathrm{i})$ & $47,7 \mathrm{ab}$ & $46,1 \mathrm{a}$ & $52,5 b$ & $66,7 \mathrm{c}$ & $51,3 a b$ & $50,7 \mathrm{~b}$ & $36,3 \mathrm{c}$ & $43,1 \mathrm{ac}$ & $<0,01$ & ns & $<0,01$ \\
\hline \multicolumn{12}{|l|}{ Mesures au pH standardisé } \\
\hline Temps de coagulation (min) (Rs) & $17,0 \mathrm{a}$ & $16,1 \mathrm{a}$ & $13,3 \mathrm{~b}$ & $12,2 \mathrm{~b}$ & 17,7 & 17,1 & 16,9 & 16,1 & $<0,01$ & ns & ns \\
\hline Temps de raffermissement (min) (K20s) & $6,6 \mathrm{a}$ & $5,6 \mathrm{a}$ & $4,3 b$ & $2,6 \mathrm{c}$ & $8,4 \mathrm{a}$ & $7,0 \mathrm{a}$ & $13,8 \mathrm{~b}$ & $8,7 \mathrm{ab}$ & $<0,01$ & $<0,1$ & $<0,01$ \\
\hline Fermeté du gel (mm) (A30s) & $54,5 \mathrm{a}$ & $56,1 \mathrm{a}$ & $59,8 \mathrm{~b}$ & $67,6 \mathrm{c}$ & $50,1 \mathrm{a}$ & $52,9 \mathrm{a}$ & $39,8 b$ & $48,7 \mathrm{ab}$ & $<0,01$ & $<0,1$ & $<0,01$ \\
\hline
\end{tabular}




\section{pH et caractéristiques de la coagulation}

Le $\mathrm{pH}$ du lait a peu varié au cours des trois premières périodes (tableau II) ; il s'est ensuite effondré pour atteindre, à la période 4 , une valeur moyenne tout à fait inhabituelle $(6,22)$. Le temps de coagulation (Ri) et le temps de raffermissement (K20i) mesurés au $\mathrm{pH}$ natif ont peu évolué au cours des deux premières périodes. Ils ont ensuite diminué d'un facteur proche de 3 au cours des deux dernières périodes, tandis que la fermeté du gel s'accroissait d'environ $40 \%$. Il est cependant à noter qu'en période 4 , le lait de la vache dont la teneur en IgG1 était de $62 \mathrm{~g} / \mathrm{kg}$ n'a coagulé qu'après un délai de 54 minuts (contre 8 minutes en moyenne pour les autres vaches), malgré un faible $\mathrm{pH}$ $(6,53)$. Ces évolutions d'une période à la suivante ont été atténuées lorsque les. mesures ont été faites après ajustement du lait au $\mathrm{pH}$ de 6,6. Le pH n'a pas été lié à la concentration des acides gras libres (26 heures).

Au début de la lactation suivante, les évolutions entre les périodes 5 et 6 ont été inverses de celles observées en fin de gestation. Le lait sécrété par les vaches du lot NT a eu tendance à présenter un temps de raffermissement plus court et une fermeté du gel plus élevée $(\mathrm{p}<0,10 ;$ mesure au $\mathrm{pH}$ standardisé de 6,60).

Pour l'ensemble des données des six périodes, le temps de coagulation (R) a été lié au $\mathrm{pH}$, et le temps de raffermissement (K20) et la fermeté du gel (A30) ont été respectivement négativement et positivement liés à la teneur en caséines (fig 2).

\section{DISCUSSION}

Du fait des contraintes d'élevage, l'alimentation des vaches a changé au cours de la fin de la gestation (pâturage puis ensilage d'herbe ; cf « Matériel et méthodes ») à un stade de gestation variable selon les ani-
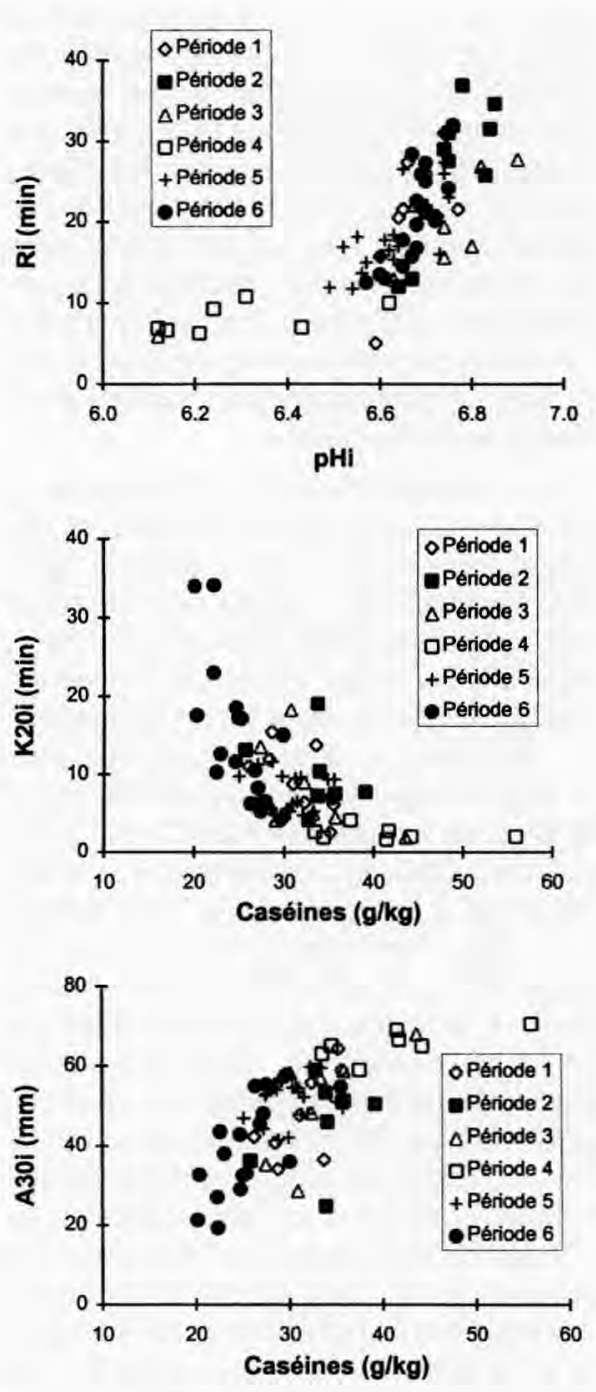

Fig 2. Liaisons entre quelques caractéristiques du lait et les paramètres de la coagulation. Périodes : $1:<-50$ jours avant le vêlage $; 2$ : -50 à -31 jours ; $3:-30$ à -11 jours ; $4:-10$ jours au vêlage ; $5: 6$ à 18 jours après le vêlage ; $6: 57$ à 82 jours.

Relationship between some characteristics of milk and its clotting properties. Periods : 1 : $<-50$ days before calving ; $2:-50$ to -31 days ; $3:-30$ to -11 days ; $4:-10$ days to calving ; $5: 6$ to 18 days after calving ; $6: 57$ to 82 days. 
maux, mais au moins 3 semaines avant le vêlage. La rentrée à l'étable a pu accélérer la diminution de la sécrétion de lait, comme l'ont montré Coulon et al (1989), avec des vaches plus productrices que les nôtres $(18 \mathrm{~kg} / \mathrm{j})$; mais, selon les résultats de ces mêmes auteurs, elle a vraisemblablement peu affecté les teneurs en matières grasses et en protéines. De plus, la forte amplitude des variations que nous avons enregistrées rend de faible importance le biais éventuel dû au changement alimentaire.

Les évolutions de la quantité de lait sécrétée et de sa composition, que nous avons enregistrées au cours des 2 derniers mois de la lactation, sont en accord avec des résultats antérieurs (Wheelock et al, 1965 ; Rémond et al, 1992 ; Rémond et Bonnefoy, 1997). Elles prolongent, en les accentuant, les variations qui se manifestent déjà à partir du milieu de la lactation ou en toute fin de la lactation « normale » (IgG1, proportion des caséines dans les protéines). Ces variations sont la conséquence de l'avancement de la gestation et de la diminution de la quantité de lait sécrétée, comme le montrent : 1) la comparaison des performances de vaches en lactation, gravides ou non : Coulon et al (1996) ont montré qu'à partir de la $20^{\mathrm{e}}$ semaine de gestation la quantité de lait sécrétée par les premières fléchissait par rapport à celle des secondes, mais que sa richesse en matières grasses et en protéines augmentait ; 2) les effets d'injections d'œstrogènes éventuellement associés à de la progestérone. Chez des vaches ou des chèvres en lactation, de telles injections ont fortement diminué la quantité de lait sécrétée mais accru ses teneurs en matières grasses et en protéines et diminué sa teneur en lactose (Peaker et Linzell, 1974 ; Mollett et al, 1976). Chez des vaches taries, des injections d'cestrogènes pendant 7 jours ont provoqué la sécrétion d'un liquide semblable au colostrum pour la formation duquel la mamelle avait sélectivement prélevé les IgG1 sanguines, comparativement aux IgG2 et à l'albumine sérique (Smith et al, 1971).
Les concentrations plus élevées des IgG1 aux périodes 3 et 4 résultent aussi de leur dilution plus faible dans un volume de lait sécrété réduit (Guy et al, 1994).

L'augmentation de la teneur en acides gras libres dans le lait natif (AGL-t0) prolonge celle d'amplitude beaucoup plus réduite observée au cours de la lactation, et semble principalement imputable au stade de gestation et au plus faible niveau de production laitière (Chazal et Chilliard, 1986 et 1987 ; Bachman et al, 1988). Consécutivement à des injections d'œstrogènes et de progestérone pendant 7 jours, Bachman (1982) a observé que l'activité lipasique totale du lait n'était pas modifiée mais que celle de la fraction grasse était accrue de $200 \%$. Après une seule injection d'oestradiol, Cartier et Chilliard (1994) ont observé que la teneur en AGL natifs était multipliée par 2,7 et que la lipolyse après $22 \mathrm{~h}$ au réfrigérateur l'était par 15. Cet accroissement de la lipolyse en fin de gestation ou consécutif à une injection d'oestradiol semblait dû à l'apparition d'une lipase sensible aux sels biliaires (Chilliard et al, 1989 ; Cartier et Chilliard, 1994).

La forte réduction de la quantité de lait sécrétée au cours de la lactation qui suit une lactation dont la période sèche a été omise est bien connue (Klein et Woodward, 1943; Rémond et al, 1992). Elle constituait, avant la mise en place des quotas laitiers, le désavantage majeur de l'omission de la période sèche. La diminution de la quantité de protéines sécrétée qui en résulte est en partie compensée par une forte augmentation (3 à $4 \mathrm{~g} / \mathrm{kg}$ ) de leur concentration, sans modification du rapport caséines/protéines, comme nous l'avions déjà observé (Rémond et al, 1992).

Malgré sa mesure retardée d'environ 24 heures, le $\mathrm{pH}$ du lait a présenté des valeurs comparables à celles couramment observées, sauf à la période $4(6,22)$. Cette dernière valeur est proche de celle $(6,4)$ enregistrée 3 à 5 jours après le vêlage par 
Grandison et al (1984). Ces auteurs avaient calculé que le $\mathrm{pH}$ au cours des 2 premiers mois de lactation était lié le plus étroitement, parmi divers constituants, à la teneur en magnésium $(r=-0,789)$ et en citrate $(r=-0,562)$ tout en précisant qu'il est difficile de distinguer les relations fonctionnelles de celles qui résultent d'une simple variation concomitante des constituants. Dans notre essai, le pH n'a pas été lié à la concentration des AGL-t26, contrairement à une hypothèse formulée par Martin et Coulon (1995).

L'évolution des paramètres de la coagulation a présenté une certaine symétrie par rapport au vêlage. Les relations observées entre le temps de coagulation ( $\mathrm{R}$ ) et le $\mathrm{pH}$ d'une part, et le temps de raffermissement (K20) ou la fermeté du gel (A30) et la teneur en caséines d'autre part ne distinguent pas les laits de fin de gestation des autres laits. Ces relations avaient déjà été observées dans des plages de $\mathrm{pH}$ plus élevées et de teneurs en caséines plus faibles (Remeuf et al, 1991 ; Machebœuf et al, 1993 ; Martin et Coulon, 1995). Le lait sécrété pendant la fin de la gestation présente donc des caractéristiques de coagulation améliorées par rapport au lait produit pendant la phase classique de la lactation, sauf lorsque la teneur en immunoglobulines est exceptionnellement élevée (cf ci-avant). La coagulation n'est qu'une composante de l'aptitude fromagère et il resterait en particulier à savoir si l'aptitude du caillé à l'égouttage n'est pas altérée par la teneur accrue en matières grasses (cf revue de Pearse et Mackinlay, 1989) qui est souvent observée (Rémond et al, 1992 ; Rémond et Bonnefoy, 1997). Par ailleurs le lait sécrété en fin de gestation contient davantage d'AGL, une lipase particulière, et un nombre plus élevé de cellules, dont les conséquences sur la qualité des produits laitiers nécessitent d'être évaluées (goût de rance...). Il est cependant à remarquer que ces modifications sont d'amplitude réduite avant le dernier mois de gestation, voire avant les 2 dernières semaines. Sous réserve que la qualité des produits laitiers ne soit pas altérée, ces résultats permettent d'envisager un raccourcissement sensible de la durée de la période sèche.

\section{REMERCIEMENTS}

Nous remercions l'équipe des techniciens animaliers du Domaine INRA d'Orcival pour leurs soins aux animaux, JC Bonnefoy, J Fléchet, $\mathrm{R}$ Lefaivre et $\mathrm{D}$ Levieux pour les analyses chimiques, et $\mathrm{C}$ Durier pour ses conseils dans les analyses statistiques.

\section{RÉFÉRENCES}

Bachman KC (1982) Effect of exogenous estradiol and progesterone upon lipase activity and spontaneous lipolysis in bovine milk. I Dairy Sci $65,907-914$

Bachman KC, Hayen MJ, Morse D, Wilcox CJ (1988) Effect of pregnancy, milk yield, and somatic cell count on bovine milk fat hydrolysis. J Dairy Sci $71,925-931$

Cartier P, Chilliard Y (1994) Effects of estradiol injection on milk composition, milk fat lipolysis and lipase activity in the non-pregnant dairy cow. Ann Zootech 43 (suppl 1), 45 s (abstr)

Chazal MP, Chilliard Y (1986) Effect of stage of lactation, stage of pregnancy, milk yicld and herd management on seasonal variation in spontaneous lipolysis in bovine milk. J Dairy Res 53, 529-538

Chazal MP, Chilliard Y (1987) Les variations individuelles de la lipolyse spontanée du lait de vache : effet du numéro de lactation et répétabilité au cours de deux lactations consécutives. Lait 67, 437-450

Chilliard Y, Cartier P, Rémond B, Fléchet J (1989) Suppression du tarissement chez les vaches laitières : B. Effets sur la lipolyse et les activités lipasiques du lait à la fin de la gestation et au début de la lactation. Compte rendu de I'AIP INRA «Qualité du lait », 15 pages

Coulon JB, D'Hour P, Rouel J (1989) Évolution de la production laitière à la rentrée à l'étable : influence de la nature de la ration de base et de la méthode de complémentation en aliment concentré. Ann Zootech $38,121-128$

Coulon JB, Rémond B, Pérochon L (1996) The effect of pregnancy on lactation persistency and milk composition in dairy cows. Anim Sci 62, 621 (Abstr)

Grandison AS, Ford GD, Millard D, Owen AJ (1984) Chemical composition and coagulating properties of renneted milks from cows during early lactation. J Dairy Res 51, 407-416 
Guy MA, McFadden TB, Cockrell DC, Besser TE (1994) Effects of unilateral prepartum milking on concentrations of immunoglobulin G1 and prolactin in colostrum. J Dairy Sci 77, 3584-3591

Klein JW, Woodward TE (1943) Influence of length of dry period upon the quantity of milk produced in the subsequent lactation. J Dairy Sci 26, 705-713

Levieux D (1991) Dosage des IgG du lait de vache par immunodiffusion radiale semi-automatisée, pour la détection du colostrum, des laits de mammites ou de fin de gestation. Mise au point du dosage. Lait 71, 327-338

Machebœuf D, Coulon JB, D'Hour P (1993) Effect of breed, protein genetic variants and feeding on cows'milk coagulation properties. J Dairy Res 60 , 43-54

Martin B, Coulon JB (1995) Facteurs de production du lait et caractéristiques des fromages. I. Influence des facteurs de production sur l'aptitude à la coagulation des laits de troupeaux. Lait 75, 61-80

Mollett TA, Erb RE, Monk EL, Malven PV (1976) Changes in estrogen, progesterone, prolactin and lactation traits associated with injection of estradiol-17 and progesterone into lactationg cows. J Anim Sci 42, 655-663

Peaker M, Linzell JL (1974) The effects of oestrus and exogenous oestrogens on milk secretion in the goat. $J$ Endocrinol 61, 231-240

Pearse MJ, Mackinlay AG (1989) Biochemical aspects of syneresis : a review. J Dairy Sci 72, 1401-1407
Remeuf F, Cossin V, Dervin C, Lenoir J, Tomassone R (1991) Relations entre les caractères physico-chimiques des laits et leur aptitude fromagère. Lait $71,397-421$

Rémond B (1987) Influence du stade de lactation et de l'âge sur la composition du lait. In : Le lait matière première de l'industrie laitière, Inra Publications, Versailles, 151-159

Rémond B, Bonnefoy JC (1997) Performance of a herd of Holstein cows managed without the dry period. Ann Zootech 46, 3-12

Rémond B, Ollier A, Miranda G (1992) Milking of cows in late pregnancy : milk production during this period and during the succeeding lactation. J Dairy Res 59, 233-241

Rowland SJ (1938) The determination of the nitrogen distribution in milk. J Dairy Res 9, 42-46

SAS (1992) SAS/STAT software : changes and enhancements, release 6.07, Cary, NC, États-Unis

Smith KL, Muir LA, Ferguson LC, Conrad HR (1971) Selective transport of IgG1 into the mammary gland : role of estrogen and progesterone. J Dairy Sci 54, 1886-1894

Swanson EW (1965) Comparing continuous milking with sixty-day dry periods in successive lactation. J Dairy Sci 48, 1205-1209

Wheelock JV, Rook JAF, Dodd FH (1965) The effect of milking throughout the whole of pregnancy on the composition of cows'milk. J Dairy Res 32, 249254 